Published in final edited form as:

Curr Treat Options Oncol. ; 19(10): 48. doi:10.1007/s11864-018-0566-5.

\title{
Harnessing the Immune System in Pancreatic Cancer
}

\author{
Satya Das, MD ${ }^{\star}$, Jordan Berlin, MD, and Dana Cardin, MD, MSCI \\ Division of Hematology and Oncology, Department of Internal Medicine, Vanderbilt University \\ Medical Center, 2220 Pierce Avenue, 777 Preston Research Building, Nashville, TN, 37232, USA
}

\section{Opinion statement}

Managing patients with metastatic pancreatic adenocarcinoma (mPDA) is a challenging proposition for any treating oncologist. Although the potency of first-line therapies has improved with the approvals of FOLFIRINOX and gemcitabine plus nab-paclitaxel, many patients are unable to derive significant benefit from later lines of therapy upon progression. Enrollment on clinical trials remains among the best options for patients with mPDA in all lines of therapy. At our institution, we routinely check for microsatellite instability (MSI-H) and perform nextgeneration sequencing (NGS) at the time of diagnosis in all good performance status mPDA patients. Although MSI-H status is only found in $1 \%$ of patients with mPDA, given pembrolizumab's tissue-agnostic approval for MSI-H tumors in later-line settings, it is a viable option when deciding on subsequent lines of therapy. Any use of immune therapy in mPDA is investigational outside the MSI-H setting. NGS can identify BRCA or other DNA damage response (DDR) defects in patients which can predict sensitivity to platinum-based therapies and influence choice of both initial and later lines of therapy. It can also identify rare actionable genomic alterations such as HER2 (2\%) and TRK fusions (0.1\%) and offer patients the option of enrollment on clinical trials with agents targeting these or other identified alterations. We believe enrolling mPDA patients on clinical trials with immune-modulating agents is critical to determine if there are other patient subsets, outside of the MSI-H setting, who would benefit from these approaches. Immunotherapy's general tolerability and potential to generate durable responses make it particularly appealing for mPDA patients. Although single-modality immunotherapy such as checkpoint inhibitors or vaccines have not demonstrated efficacy in this disease, combinatorial strategies targeting unique aspects of PDA including the tumor micro-environment and desmoplastic stroma have shown preclinical or early-phase success. Validating these treatments with later-phase prospective studies is essential to making immunotherapy a routine component of the treatment armamentarium for mPDA patients.

* satya.das@vumc.org.

This article is part of the Topical Collection on Upper Gastrointestinal Cancers

Conflict of Interest

Satya Das has received compensation from Targeted Oncology for service as a consultant (Clinical Congress Consultants).

Dana B. Cardin has received research funding from Celgene, EMD Serono, Hoffman-La Roche, Incyte, Oncolytics Biotech, Synta

Pharmaceuticals, Bristol-Myers Squibb, Advaxis, and Lilly, and has received compensation from Cornerstone Pharmaceuticals, Rafael

Pharmaceuticals, and AbbVie for service as a consultant.

Jordan Berlin has received research funding from Novartis, AbbVie, Immunomedics, Taiho, Genentech/Roche, Bayer, Incyte, Pharmacyclics, Five Prime, EMD Serono, and Loxo.

Human and Animal Rights and Informed Consent

This article does not contain any studies with human or animal subjects performed by any of the authors. 


\section{Keywords}

Metastatic pancreatic cancer; Immunotherapy; Microsatellite instability; Tumor microenvironment

\section{Introduction}

Metastatic pancreatic adenocarcinoma (mPDA) remains a devastating disease with 5-year survival rates of $<5 \%$ [1]. Although combinatorial chemotherapy approaches are initially effective, patients typically progress in months. Median PFS and OS with second-line chemotherapy regimens, after failure of gemcitabine- or 5-FU-based regimens, range from $0.9-5.1$ to $1.3-8.5$ months, respectively [2]. Patients often decline precipitously, and historically, only $17-45 \%$ of patients receive therapy beyond the first line [3, 4]. As such, tolerable and effective later-line treatments remain an area of great need in this disease. Immunotherapy's general tolerability and potential to generate durable (and sometimes complete) responses make it a natural fit in this setting; however, its benefit has yet to be realized in PDA cancer patients. Some of the intrinsic characteristics of PDA that preclude immune responsiveness include low somatic mutational burden, minimal neo-epitope presentation, and an immunosuppressive tumor microenvironment (TME). The TME is characterized by abundant tumor-associated macrophages (TAMs)/regulatory CD4 T-cells (Treg)/myeloid-derived suppressor cells (MDSCs), a dense desmoplastic stroma, and scarce activated cytotoxic CD8 T-cells [5, 6••, 7] (Fig. 1). Given these characteristics, it is not surprising that single-agent therapy, with checkpoint inhibitors targeting PD1/PDL1 or vaccines, has demonstrated limited benefit in this disease [8••, 9]. In the subsequent paragraphs, we will discuss some of the ongoing novel combinatorial approaches, seeking to transform the "cold" TME into an active one in mPDA patients.

\section{Adding to checkpoint inhibitors}

- Although single-agent checkpoint inhibitor activity in mPDA patients, outside of the microsatellite high (MSI-H) setting, has been minimal [10••], combinatorial approaches with other immune-modulating agents, chemotherapy, vaccines, or radiation have demonstrated promise.

\section{Chemotherapy combinations}

- Weiss et al. reported findings from the metastatic PDA patient arm of the NCT02331251 trial [11]. In this phase I/II study, 17 patients were treated with the combination of gem, nab, and pembrolizumab. During dose escalation, patients were split into two cohorts, treatment naive and previously treated, due to DLT experienced in the latter. In the former cohort, the maximum tolerated dose (MTD) of the combination was pembrolizumab $2 \mathrm{mg} / \mathrm{kg}$, gem 1000 $\mathrm{mg} / \mathrm{m}^{2} \mathrm{D} 1$ and $\mathrm{D} 8$, and nab $125 \mathrm{mg} / \mathrm{m}^{2} \mathrm{D} 1$ and D8 every 21 days. While the study did not meet its phase II endpoint of 15\% complete response (CR) (by iRECIST), among 11 patients evaluable for response, DCR was 100\%. Median PFS and OS were 9.1 and 15 months for treatment-naive PDA patients.

- Wainberg et al. published preliminary results from a phase I study of nivolumab plus gemcitabine (gem) and nab-paclitaxel (nab) in metastatic PDA patients [12]. In arm A of the 
study, 11 patients who had received one prior line of therapy received nab $125 \mathrm{mg} / \mathrm{m}^{2} \mathrm{D} 1$, D8, and D15 and nivolumab $3 \mathrm{mg} / \mathrm{kg}$ D1 and D15 every 28-day cycle. In arm B of the study, six treatment-naive patients received the regimen in arm A plus gem $1000 \mathrm{mg} / \mathrm{m}^{2} \mathrm{D} 1, \mathrm{D} 8$, and D15 every 28 days. No dose-limiting toxicities (DLTs) were seen in arm A and only one (hepatitis) was seen in arm B. Disease control rate (DCR) was achieved in 55\% of patients in arm A and $100 \%$ of patients in arm B. Median treatment duration was 12.6 and 15.5 weeks for arms A and B, respectively. Patients are continuing enrollment on part B of this study.

- In preclinical models, CD40 agonists such as APX005M bind to the CD40 receptor (part of TNF receptor family) on antigen-presenting cells and B-cells as well as activated CD4 and CD8 T-cells [13]. These compounds cause tumor regression in both T-cell-independent, largely through macrophage reprogramming to a TH1 phenotype, and T-cell-dependent manners. Prior work with CD-40 agonists such as CP-870, 893 was published by Beatty et al. [14]. In a two-center study, mPDA patients were treated with standard dose gem or standard dose gem plus CP-870, 8932 days after the first dose of gemcitabine with each cycle. Four of 21 patients achieved PR, and 11 achieved stable disease as their best response for a DCR of 71.4\%. Median progression-free survival (PFS) was 5.2 months, and median overall survival (OS) was 8.4 months. A collaborative effort between the Parker Institute and Cancer Research Institute has led to a phase I/II randomized trial of gem, nab, and nivolumab with or without the CD40 agonist monoclonal antibody APX005M in previously treated mPDA patients [NCT03214250].

\section{Immunotherapy combinations}

- MORPHEUS-PDAC is a phase I/II platform study randomizing progressive second-line mPDA patients to a variety of combined immunotherapy approaches or chemotherapy (gem nab or FOLFOX) [15••]. Some of the combination arms include atezolizumab plus the MEK inhibitor cobimetinib, atezolizumab plus the hyaluronidase inhibitor PEGPH20, or atezolizumab plus the CXCR4 inhibitor BL-8040.

- Abraham et al. demonstrated the preclinical ability of BL-8040 to increase CD8/CD4 Tcell infiltration and dendritic cell mobilization in multiple murine cancer cell line models [16]. In the PDA murine model, BL-8040 increased CD8 cell accumulation within the tumor and suppressed growth.

- Hidalgo et al. presented interim pharmacodynamic data from the monotherapy portion of phase II COMBAT study where mPDA patients were treated with 5 days of BL-8040 leadin, followed by 21-day cycles where patients received pembrolizumab D1 and BL-8040 thrice weekly [17]. Blood samples were obtained on D1 and D5 for flow cytometric analysis while liver biopsies, when available, were assessed to characterize dynamic changes in the nature of the immune cell infiltrate. Flow cytometry revealed a 2 -fold reduction in Treg levels and no change in levels CD8 T-cells or NK cells from peripheral blood. Liver biopsies from 7 available patients revealed up to a 15-fold increase in CD3-positive cells and 2-fold increase in CD8 T-cells in the TME in 43\% of patient samples, demonstrating the proof-ofprinciple mechanism of the CD40 agonist. The study is ongoing. 
- O'Reilly et al. reported findings from the ALPS study where 65 second-line mPDA patients, post-gemcitabine (gem)- or flurouracil (5-FU)-based regimens, were randomized to monotherapy with the PD-L1 inhibitor durvalumab (1.5 g IV Q4 weeks) or the combination of durvalumab (1.5 g IV Q4 weeks) plus the CTLA-4 inhibitor tremelimumab (75 mg IV Q4 weeks) [18]. Part A was a safety lead-in while part B was intended to assess efficacy by overall response rate (ORR) if a minimum efficacy signal was demonstrated in part A. In the first part of the study, the most common grade 3 treatment-related adverse events (TRAEs) were diarrhea $(9.4 \%)$ and fatigue $(6.3 \%)$ in the combination arm and ascites $(3.1 \%)$ and hepatitis (3.1\%) and lipase elevation (3.1\%) in the mono-therapy arm. One patient in the combination arm (3.1\%) demonstrated a partial response (PR) which persisted $>12$ months while $9.4 \%$ of patients demonstrated disease control. In the durvalumab-alone arm, $6.1 \%$ patients demonstrated PR and disease control. Given the lack of efficacy signal seen in part A, part B was not pursued.

\section{Vaccines}

- Single-agent vaccine studies in mPDA patients have demonstrated mixed results. Some, like the recombinant virus-based MUC- 1 and CEA targeting vaccine studies were negative, while others such as such as the combination of CRS-207 (attenuated listeria vector expressing mesothelin) and GVAX (irradiated GM-CSF-secreting allogeneic pancreatic cancer cells)/cyclophosphamide (Cy) and gastrin-based vaccine studies have demonstrated activity [19-21].

- Building upon the hints of efficacy demonstrated from the combination of CRS-207 and GVAX/Cy, Le et al. assessed the triplet therapy with these vaccines and nivolumab in PDA patients in the STELLAR study [22•]. In this phase II trial, metastatic second-line patients were randomized 1:1 to receive 2 cycles of GVAX/Cy and nivolumab followed by 4 doses of nivolumab/CRS-207 or 2 doses of GVAX/Cy and 4 doses of CRS-207. The primary endpoint of this study is overall survival and results are pending. A later randomized study of GVAX/Cy and CRS-207 versus physician's choice single-agent chemotherapy in secondline mPDA patients failed to demonstrate OS benefit of the combination vaccines compared to chemotherapy [23].

- The combination of GVAX and ipilimumab was explored in a phase Ib study by Le et al. [24]. Thirty patients with advanced or mPDA, who had previously received gemcitabine chemotherapy, were randomized to ipilimumab alone (10 mg/kg Q3 weeks, then Q12 weeks) or ipiliumumab (at same dose) plus GVAX $\left(2.5 \times 10^{8}\right.$ cells $\times 2$, administered on weeks 1,4 , 7 , and 10). Primary objective was to demonstrate safety of the combination with secondary endpoints of OS and DCR. Three G3/G4 AE were noted in both arm 1 and arm 2 (2 total instances of colitis). Median OS was 4.3 and 5.7 months for arm 1 and arm 2, respectively (HR $0.51, p=.07$ ). Patients with a greater diversity of mesothelin-directed T-cell response demonstrated longer survival.

- Based on these findings, there is an ongoing randomized phase 2 study of nivolumab, ipilimumab and CRS-207, with or without GVAX, in previously treated mPDA patients [NCT03190265]. Patients will receive nivolumab $360 \mathrm{mg}$ Q3 weeks, ipilimumab $1 \mathrm{mg} / \mathrm{kg}$ 
Q3 weeks, CRS-207 Q3 weeks, and potentially GVAX/Cy Q3 weeks (for 6 treatments). Primary endpoint of the study is ORR.

\section{Radiation, epigenetics, vitamin D}

- Preclinical evidence across tumor types suggests that HDAC inhibition augments MHC class I presentation, decreases PDL1 and PDL2 expression, and decreases ornithine decarboxylase activity, creating a potential synergistic opportunity to combine it with checkpoint inhibitors [25]. The HDAC I and III inhibitor entinostat is being combined with nivolumab in an ongoing phase II study in patients with advanced cholangiocarcinoma and PDA [NCT03250273].

- Investigators leading the RAD VAX trial from the University of Pennsylvania are seeking to assess whether the abscopal effect, a phenomenon where local radiation is associated with regression of metastatic cancer distant from the radiated area, can be exploited in mPDA and other advanced malignancy patients [26]. Patients in cohort 1 of the study will receive 24 Gy in 3 fractions of stereotactic radiation (during week 1), durvalumab $20 \mathrm{mg} / \mathrm{kg}$ and tremelilumab $1 \mathrm{mg} / \mathrm{kg}$ every 4 weeks for 4 doses, followed by durvalumab $10 \mathrm{mg} / \mathrm{kg}$ every 2 weeks for 18 doses. Patients in cohort 2 of the study will receive $17 \mathrm{~Gy}$ in one fraction of stereotactic radiation (during week 1) along with durvalumab and tremelilumab at the same dosing schedule described above. Cases of tumor regression in mPDA patients receiving XRT to progressive sites along with simultaneous G-CSF have been described [27].

- Vitamin D receptor agonists have demonstrated an ability to sensitize PDA lesions to checkpoint blockade through reducing MDSC and TRegs [28]. Based on this finding, Chung et al. have designed the SU2C catalyst trial [29]. In this phase II study, first-line mPDA patients who have achieved SD or PR for 2 months after standard first-line chemotherapy, with a serum vitamin D level $<50 \mathrm{ng} / \mathrm{ml}$, are randomized 1:1 to pembrolizumab $200 \mathrm{mg}$ every 3 weeks plus paracalcitriol $25 \mathrm{mcg}$ thrice weekly or pembrolizumab plus placebo. The primary endpoint of the study is to assess the 6-month progression-free survival of patients using the maintenance strategy. Borazanci et al. reported promising findings from a phase II pilot study of nivolumab, nab-paclitaxel, paracalcitriol, gemcitabine, and cisplatin in treatment-naive mPDA patients [30]. Patients on treatment receive nivolumab $240 \mathrm{mg}$ D1, D15, and D29; nabpaclitaxel $125 \mathrm{mg} / \mathrm{m}^{2}$ plus gemcitabine $1000 \mathrm{mg} / \mathrm{m}^{2}$ plus cisplatin 25 $\mathrm{mg} / \mathrm{m}^{2} \mathrm{D} 1, \mathrm{D} 8$, D22, and D29; and paracalcitriol $25 \mu \mathrm{g}$ twice weekly on 42-day cycles. The primary objective of the study is to demonstrate efficacy through measuring ORR, PFS, and OS. Ten patients have been treated thus far, with eight patients achieving PR (80\% ORR) and two achieving SD (100\% DCR). Median PFS is 8.2 months and OS has yet to be reached. The most common grade 3 and 4 TRAEs are thrombocytopenia (100\%), anemia (50\%), and colitis (20\%).

\section{Transforming the TME \\ Targeting TAMs and MDSC}

- TAM and MDSC are crucial contributors to the immunosuppressive TME in PDA and are drawn to the tumor interface by high levels of colony-stimulating factor 1 (CSF-1). Elegant 
preclinical work in has demonstrated inhibiting CSF1R in PDA models reduces TAM infiltration, reprograms existing TAM to a M1 (more inflammatory, IFN-y responsive) phenotype and increases cytotoxic T-cell infiltration while reducing Treg concentrations [31]. Wainberg et al. have reported preliminary findings from the PDA dose expansion cohort of a phase I study combining the CSF-1R6 inhibitor cabiralizumab with nivolumab in progressive metastatic PDA patients [32]. Thirty-one PDA patients received the established RP2D of $4 \mathrm{mg} / \mathrm{kg}$ of cabiralizumab plus $3 \mathrm{mg} / \mathrm{kg}$ of nivolumab every 2 weeks. Four out of 31 patients demonstrated an ORR (13\%), and 3 out of the 4 continue to receive treatment with times on treatment ranging from 5.6 to 9.2 months. All four patients have microsatellite-stable (MSS) tumors. Findings from this study have led to NCT NCT03336216, a randomized phase II study of cabralizumab and nivolumab alone versus the combination with FOLFOX versus the combination with gem and nab.

- There is also an ongoing phase Ib/II study of the CSF-1R targeting monoclonal antibody lacnotuzumab with the PD-1 inhibitor spartalizumab in patients with advanced PDA, melanoma, endometrial cancer, or tripe-negative breast cancer [33]. Thirty of the 48 patients who received treatment had mPDA. Across the whole cohort, DCR was $21 \%$ (1 PR) by RECIST criteria and 27\% (13 out of 48) by iRECIST; 6 of the 13 patients with DCR had PDA. Three of these 6 PDA patients had disease control for $>300$ days.

- Chemokine C-C ligand 2 (CCL2) and chemokine C-C ligand 5 (CCL5) are cytokines overexpressed in PDA, which play important roles in the influx of TAM and Treg into the TME. CCL2-positive TAM comprises close to $30 \%$ of the tumor-infiltrating lymphocytes (TILs) in the TME of PDA patients and vastly outnumbers cytotoxic T-cells [34•]. In murine models of PDA treated with an anti-CCR2 (the receptor of CCL2) agent, markedly less TAM infiltration and greater cytotoxic T-cell infiltration was appreciated. Tumors from antiCCR2-treated mice also demonstrated a gene expression profile shift from Th2 to Th1, characterized by an increase in IFN-Y-mediated signaling [35]. In CCL5 knockout murine PDA models, 50\% less TReg were noted in harvested tumors compared to tumors from WT models. The CCR2/CCR5 antagonist BMS-813160 is being trialed in combination with nivolumab or gem nab in metastatic pancreatic cancer patients [NCT03184870].

\section{Altering the desmoplastic stroma}

- Focal adhesion kinase (FAK) is a non-receptor cytosolic tyrosine kinase, often overexpressed in PDA, which signals to elements in the extracellular matrix and promotes cell motility, migration, proliferation, and invasion. It also plays a role in tumor-associated fibrosis and recruitment of regulatory immune cells. Preclinical data suggests potential mechanistic synergy between FAK inhibitors and PD-1 checkpoint blockers [36]. Gilliam et al. have published data from a phase I study exploring the combination of the FAK inhibitor defactinib with pembrolizumab and gemcitabine [37]. Dose escalation has been completed, and the RP2D has been determined to be defactinib $400 \mathrm{mg}$ oral twice daily, pembrolizumab $200 \mathrm{mg}$ (D1) and gemcitabine $1000 \mathrm{mg} / \mathrm{m}^{2}$ (D1 and D8) every 21 days. Among 13 patients evaluable for a treatment response, 7 have demonstrated stable disease (SD) with a median time-on-treatment of 4.2 months. 
- A promising triplet combination with the dipeptidyl peptidase (DPP) 8/9 inhibitor BXCL701, CD122 agonist NKTR-214, and PD-1 inhibitor in a murine PDA model were recently presented by Rastelli et al. [38]. By targeting DPP 8/9, BXCL701 inhibits fibroblast-associated protein (FAP), a key activator of cancer-associated fibroblasts (CAFs) which contribute to generation of a fibrotic barrier and whose expression has been associated with poor prognosis in PDA patients [39]. The NKTR-214 compound activates and selectively expands T and NK cell populations through IL-2 signaling. Mice bearing Pan02 tumors were treated with the triplet combination. One hundred percent of the mice (9/9) became tumor free by day 21 and remained tumor free until day 60. Six of these nine mice were re-challenged with new tumor cells and five of the six rejected them. IHC from tumors of treated animals sacrificed at D3 revealed significantly decreased FAP levels, increased peri-tumoral T-cells, and increased inflammatory cytokines (TNF-alpha, IFN-y).

- The BTK inhibitor ibrutinib demonstrated anti-tumor effect in murine PDA models in part due to its ability to affect TME immunomodulation. Some of its modulating effects include decreasing tumor-associated inflammation and desmoplasia, increasing CD8 T-cell infiltration, and shifting TAM to a Th1 phenotype [40]. The RESOLVE study, introduced by Tempero et al., is a randomized phase II/III study exploring the addition of ibrutinib to gem nab in first-line mPDA patients [41]. In this study, mPDA patients will receive ibrutinib 560 mg oral daily, or matched placebo with standard dosing of gem nab, with a primary endpoint of PFS. Enrollment is ongoing.

- Given the previously noted preclinical data with ibrutinib, it was explored in combination with the PDL1 inhibitor durvalumab in a phase Ib/II multicenter study. From Hong et al., the RP2D was $560 \mathrm{mg}$ oral daily ibrutinib and durvalumab $10 \mathrm{mg} / \mathrm{kg}$ every 2 weeks [42]. Fortynine out of 122 evaluated patients had second-line mPDA. ORR was $2 \%$ in these patients with 1 PR (duration 10 months). Median OS in the PDA patient cohort was 4 months.

- Nearly 50\% of PDA patients possess mutations in SMAD4, a central component in TGFB-mediated signaling [43]. Preclinical models suggest that inhibiting the TGF-B pathway through ligand sequestering with monoclonal antibodies or receptor inhibition with small molecule inhibitors can reduce metastatic progression, fibroblast deposition, and avoidance of tumor apoptosis [44]. Melisi et al. randomized unresectable stage II-IV PDA patients in a 2:1 fashion to the TGF-B receptor inhibitor galunisertib (150 mg BID for 14 days on, 14 days off) plus gemcitabine or gemcitabine plus placebo in a phase II study [45]. The primary endpoint of the study was OS. Median OS in the experimental arm was 9.1 months compared to 7.59 months in the control arm (HR 0.8, $p=.084$ ). In the subgroup of patients with TGF-B levels $<4224 \mathrm{pg} / \mathrm{mL}$, median OS was 10.9 months with the TGF-B inhibitor compared to 7.2 months in the control arm (HR 0.64, $p=.076$ ). Median PFS reached a statistically significant difference between the two arms. Galunisertib is being explored in a phase I study in combination with durvalumab in mPDA patients [NCT02734160].

\section{Activating T-cells}

- IL-10 is a cytokine which induces STAT-3-mediated proliferation and activity of CD8 Tcells. Single-agent activity of AM0010 (pegylated IL-10) was demonstrated in mPDA patients as a salvage therapy [46]. Given the preclinical synergy with oxaliplatin, AM0010 
plus FOLFOX was tested in second line and beyond mPDA patients in a single-arm phase II study [47]. Twenty-five patients were treated with AM0010 (5 $\mu \mathrm{g} / \mathrm{kg}$ daily) plus FOLFOX (every 14 days) with a primary endpoint of ORR. Immune biomarkers such as clonality of peripheral T-cells and tumor T-cell infiltration by whole-exome sequencing were collected. ORR in 19 evaluable patients was $15.8 \%$ with a DCR of $78.9 \%$. Median PFS was 3.5 months, median OS was 10.2 months, and 1-year survival was $43 \%$. The combination was well tolerated with most common G3/G4 TRAE being thrombocytopenia, anemia, and neutropenia. FOLFOX plus AM0010 is now being compared to FOLFOX alone in the randomized phase III Sequoia study.

- CD73, a cell surface enzyme which is upregulated in several carcinomas including PDA, generates extracellular adenosine and exerts an immunosuppressive effect on T-cells [48]. Oleclumab (MEDI9447) is a monoclonal antibody which binds to CD73 and prevents adenosine generation. It was trialed as monotherapy or in combination with durvalumab in a phase I study in advanced colorectal (CRC) and pancreatic cancer patients [49]. Oleclumab was given in escalating doses IV every 2 weeks with or without durvalumab $10 \mathrm{mg} / \mathrm{kg}$. Initial dose escalation in monotherapy and combination cohorts has been completed with no DLT. Treatment with oleclumab decreased tumor CD73 expression in 5 of 9 patients while increasing CD8 T-cells intratumorally in all samples. PR and DCR was demonstrated in 2/20 (10\%) and 4/20 (25\%) of PDA patients, respectively. Treatment for two mPDA patients is ongoing at 140 and 182 days. Dose expansion in PDA and CRC cohorts is ongoing.

- Indoleamine 2,3 dioxygenase-1 (IDO-1) is an enzyme whose immunomodulatory mechanism stems from tryptophan depletion which results in T-cell cell cycle arrest and kynurenine-mediated lympho-toxic effects [50]. Specifically, IDO1 results in T-cell and NK cell suppression, activates Tregs, and promotes immune tolerance to tumor neo-antigens. Levels of IDO-1 are disproportionately high in PDA patients compared to patients without PDA. There also appears to be a stage association as patients with more advanced disease tend to have higher levels of IDO1 than patients with earlier-stage disease [51]. After safety was demonstrated in a phase I study, the combination of the IDO1 inhibitor indoximod plus gemcitabine and nab in first-line metastatic PDA patients was evaluated in a single-arm phase II study, with results recently reported by Bahary et al. [52]. One hundred thirty-five patients started treatment and 104 were evaluable for response. ORR in this group of patients was $46.2 \%$ (45.2\% PR) with a median OS of 10.2 months. Immune biomarkers from 11 patients in the study demonstrate responding patients to have increased intratumoral CD8 Tcell density than in non-responders $(p<.03)$.

- Bispecific T-cell engaging (BITE) antibodies have demonstrated promise in advanced malignancies due to their ability to draw activated CD3-positive T-cells to a tumor-specific target. Lum et al. reported findings from five locally advanced and metastatic PDA patients treated with an anti-EGFR anti-CD3 bispecific antibody [53]. Patients received the BITE in the phase I portion of the study at escalating infusions (10 to 20 to $40 \times 10^{9}$ cells) for 3 weekly infusions and then a booster infusion 3 months later. Four patients received $10 \times$ $10^{10}$ BITE cells twice weekly for 4 weeks in the phase II portion of the study. One patient in the phase I had a PR for 6.5 months while two patients who progressed achieved CR with subsequent chemotherapy. Two patients in the phase Ib have SD after 2.3 and 21 months. 
Median OS is 31 months for all nine patients. Beyond the direct tumor-directed effects of the anti-EGFR BITE, the potential sensitization to subsequent chemotherapy is an element that needs formal evaluation. Results from the phase $1 \mathrm{~b}$ portion of the study remain pending [NCT01420874].

- Epithelial cell adhesion molecule (EpCAM) is overexpressed and altered in most epithelial tumors including PDA [54]. It frequently is expressed by tumor-initiating cancer stem cells (CSC) in the disease, offering a potential target to eliminate these notoriously chemorefractory cells. Cioffi et al. studied the ability of the EpCAM/CD3 BITE antibody MT110 to induce CD8 T-cell activation and lysis of PDA in vitro and in vivo [55]. MT110 increased apoptosis in PDA cell line models and depletion of CSC and tumor shrinkage in PDA xenograft models. There was only modest additional tumor shrinkage seen in xenografts treated with gemcitabine plus MT110. Catumaxomab is a trifunctional EpCAMdirected BITE (with a Fcy domain which initiates antibody-dependent cell-mediated cytotoxicity by binding to macrophages, dendritic cells, NK cells) whose activity in reducing malignant ascites was demonstrated based on a randomized phase II/III study [56].

- Integrating CAR T-cells into the care of refractory solid tumor patients such as those with PDA is an area of great allure. Some of the approaches that have been trialed include EGFR, HER2, mesothelin, and MUC1-directed CAR T-cells. HER2 overexpression in PDA portends a poor prognosis, and there is an ongoing anti-HER2 CAR-mediated trial in PDA patients [NCT02713984]. Mesothelin-directed CAR T-cells are also being trialed as a local infusion for unresectable PDA patients so as to increase the direct tumor effect of the therapy and minimize toxicity to normal tissue [NCT02706782]. CAR T-cells targeting MUC1 demonstrated preclinical promise in PDA xenograft models which are being explored in an ongoing trial [NCT02587689].

\section{Summary}

While initial single-agent trials of PD-1 or PDL-1 inhibitors in PDA patients have had little to no effect, major efforts are being made to incorporate immunotherapy into the care of patients with mPDA given the dramatic improvement in survival we have seen in other refractory malignancies. Even combinations of checkpoint inhibitors (PD-1 and CTLA-4) have shown limited benefit, suggesting that multimechanism approaches may be necessary to overcome the immune "cold" microenvironment. One of the primary barriers to these efforts is thought to be the immunosuppressive TME. Attacking different components of the TME simultaneously appears to be the only chance for success given the limited results with single-modality therapy. Unsurprisingly, some of the more promising approaches in PDA patients involve combinations of checkpoint-inhibiting, T-cell stimulating, chemotherapy, targeted and stromal altering agents. These approaches hold out hope because of their ability to generate increased cancer cell apoptosis and neo-epitope release, improve T-cell infiltration and stimulation, and decrease fibrosis. We may find that the optimal use of immunotherapy in PDA is in earlier, neoadjuvant and adjuvant, settings. Ongoing studies are exploring whether immunotherapy, as has been done with chemotherapy and chemoradiation, can be moved up-front. 


\section{References and Recommended Reading}

Papers of particular interest, published recently, have been highlighted as:

- Of importance

•• Of major importance

1. SEER Database 2018. https://seer.cancer.gov/statfacts/html/pancreas.html.

2. Walker E, Ko A. Beyond first-line chemotherapy for advanced pancreatic cancer: an expanding array of therapeutic options? World J Gastroenterol. 2014;20(9):2224-36. [PubMed: 24605022]

3. Van Hoff D, Ervin T, Arena F, et al. Increased survival in pancreatic cancer with nab-paclitaxel plus gemcitabine. N Engl J Med. 2013;369:1691-703. [PubMed: 24131140]

4. Nagrial A, Chin VT, Sjoquist KM, et al. Second-line treatment in inoperable pancreatic adenocarcinoma: a systematic review and synthesis of all clinical trials. Crit Rev Oncol Hematol. 2015;96:483-97. [PubMed: 26481952]

5. Liu Q, Liao Q, Zheo Y. Chemotherapy and tumor microenvironment of pancreatic cancer. Cancer Cell Int. 2017;17(68):1-17. [PubMed: 28053596]

6••. Johnson B III, Yarchoan M, Lee V, et al. Strategies for increasing pancreatic tumor immunogenicity. Clin Cancer Res. 2017;23(7):1656-69. [PubMed: 28373364] A detailed review exploring the complex layers of immuno-suppression present in the TME of PDA patients and why combinatorial approaches, targeting some of these different elements, is a necessity to making immunotherapy a viable treatment option in this disease.

7. Zhang Y, Velez-Delgado A, Li M, et al. Myeloid cells are required for PD-1/PD-L1 checkpoint activation and the establishment of an immunosuppressive environment in pancreatic cancer. Gut. 2016;66:124-36. [PubMed: 27402485]

$8 \bullet \cdot$. Royal RE, Levy C, Turner K, et al. Phase 2 trial of single agent Ipilimumab (anti-CTLA-4) for locally advanced or metastatic pancreatic adenocarcinoma. J Immunother. 2010;33:828-33. [PubMed: 20842054] One of the initial studies demonstrating that single-agent checkpoint inhibitors had very limited activity in mPDA. This demonstrated the need for combinatorial immune-modulating strategies moving forward.

9. Brahmer J, Tykodi S, Chow L, et al. Safety and activity of anti-PD-L1 antibody in patients with advanced Cancer. N Engl J Med. 2012;366:2455-65. [PubMed: 22658128]

$10 \bullet$. Le D, Durham J, Smith K, et al. Mismatch-repair deficiency predicts response of solid tumors to PD-1 blockade. Science. 2017;357(6349):409-13. [PubMed: 28596308] A seminal proof-ofprinciple study which demonstrated that MSI-H tumors have significant response rates to singleagent immunotherapy with pembrolizumab. This led to FDA approval for pembrolizumab in MSI-H tumors of any type after failure of initial-line chemotherapy.

11. Weiss GJ, Blaydorn L, Beck J, Bornemann-Kolatzki K, Urnovitz H, Schütz E, et al. Phase Ib/II study of gemcitabine, nab-paclitaxel, and pembrolizumab in metastatic pancreatic adenocarcinoma. Investig New Drugs. 2018;36(1):96-102. [PubMed: 29119276]

12. Wainberg Z, Hochster H, George B, et al. Phase I study of nivolumab (nivo) + nab-paclitaxel (nabP) \pm gemcitabine $(\mathrm{Gem})$ in solid tumors: interim results from the pancreatic cancer (PC) cohorts. J Clin Oncol. 2017;35(4):S412.

13. Beatty G, Chiorean G, Fishman M, et al. CD40 agonists alter tumor stroma and show efficacy against pancreatic carcinoma in mice and humans. Science. 2012;331(6024):1612-6.

14. Beatty G, Torigian D, Chiorean E, et al. A phase I study of an agonist CD40 monoclonal antibody (CP-870,893) in combination with gemcitabine in patients with advanced pancreatic ductal adenocarcinoma. Clin Cancer Res. 2013;19(22):6286-95. [PubMed: 23983255]

15••. Manji G, Bendell J, Oh DY, et al. MORPHEUS: a phase Ib/II multi-trial platform evaluating the efficacy and safety of cancer immunotherapy (CIT)-based combinations in patients (pts) with gastric or pancreatic cancer. J Clin Oncol. 2018;36(4):S530.An example of a platform study which will likely serve as a template for how future immune combinations are trialed in mPDA patients. 
16. Abraham M, Mishalian I, Harel Y, et al. Effect of BL-8040, high-affinity CXCR4 antagonist, on Tcell infiltration, tumor growth, and synergy with immunomodulatory agents. J Clin Oncol. 2017;35(15):S14544.

17. Hidalgo M, Epelbaum R, Semenisty V, et al. Evaluation of pharmacodynamic (PD) biomarkers in patients with metastatic pancreatic cancer treated with BL-8040, a novel CXCR4 antagonist. J Clin Oncol. 2018;36(5):S88.

18. O'Reilly E, Oh DY, Dhani N, et al. A randomized phase 2 study of durvalumab monotherapy and in combination with tremelimumab in patients with metastatic pancreatic ductal adenocarcinoma (mPDAC): ALPS study. J Clin Oncol. 2018;36(4):S217.

19. Kaufman HL, Kim-Schulze S, Manson K, et al. Poxvirus-based vaccine therapy for patients with advanced pancreatic cancer. J Transl Med. 2007;5(60):1-10. [PubMed: 17201925]

20. Gilliam AD, Broome P, Topuzov EG, Garin AM, Pulay I, Humphreys J, et al. An international multicenter randomized controlled trial of G17DT in patients with pancreatic cancer. IJ Pancreas. 2012;41(3):374-9.

21. Le DT, Wang-Gillam A, Picozzi VJ, et al. Interim safety and efficacy analysis of a phase II, randomized study of GVAX pancreas and CRS-207 immunotherapy in patients with metastatic pancreatic cancer. J Clin Oncol. 2013;31(suppl):abstr 4040.

22•. Le D, Crocenzi T, Urum J, et al. Randomized phase II study of the safety, efficacy and immune response of GVAX pancreas (with cyclophosphamide) and CRS-207 with or without nivolumab in patients with previously treated metastatic pancreatic adenocarcinoma (STELLAR). J Immunother Cancer. 2015;3(S2):P155.An important study assessing the potential mechanistic synergy between vaccines and checkpoint blockade. It highlights the pre-clinical principle that vaccination increases intra-tumoral lymphocyte influx while PD-1 inhibition maintains the activation of these cells.

23. Le D, Ko A, Wainberg Z, et al. Results from a phase $2 b$, randomized, multicenter study of GVAX pancreas and CRS-207 compared to chemotherapy in adults with previously-treated metastatic pancreatic adenocarcinoma (ECLIPSE Study). J Clin Oncol. 2017;35(4):S345.

24. Le D, Lutz E, Uram J, et al. Evaluation of Ipilimumab in combination with allogeneic pancreatic tumor cells transfected with a GM-CSF gene in previously treated pancreatic cancer. $\mathrm{J}$ Immunother. 2013;36(7):382-9. [PubMed: 23924790]

25. Chumsri S, Necela BM, Ordentlich P, et al. Immunomodulatory effects of entinostat on PD-L1 and MHC class I and II in different subtypes of breast cancer. Cancer Res. 2016;76(4):SABCS15-P204-02.

26. Postow M, Callahan M, Barker C, et al. Immunologic correlates of the abscopal effect in a patient with melanoma. N Engl J Med. 2012;366:925-31. [PubMed: 22397654]

27. Shi F, Wang X, Teng F, Kong L, Yu J. Abscopal effect of metastatic pancreatic cancer after local radiotherapy and granulocyte-macrophage colony-stimulating factor therapy. Cancer Biol Ther. 2017;18(3):137-41. [PubMed: 28267380]

28. Davis-Yadley A, Malafa M. Vitamins in pancreatic cancer: a review of underlying mechanisms and future applications. Adv Nutr. 2015;6(6):774-802. [PubMed: 26567201]

29. Chung V, Borazanci E, Jameson G. A SU2C catalyst randomized phase II trial of pembrolizumab with or without paricalcitol in patients with stage IV pancreatic cancer who have been placed in best possible response. J Clin Oncol. 2018;36(15):S4154.

30. Borazanci E, Jameson G, Borad M, et al. A phase II pilot trial of nivolumab (N) + albumin bound paclitaxel $(\mathrm{AP})+$ paricalcitol $(\mathrm{P})+$ cisplatin $(\mathrm{C})+$ gemcitabine $(\mathrm{G})(\mathrm{NAPPCG})$ in patients with previously untreated metastatic pancreatic ductal adenocarcinoma (PDAC). J Clin Oncol. 2018;36(4):S358.

31. Zhu Y, Knolhoff B, Meyer M. et al. CSF1/CSF1R blockade reprograms tumor-infiltrating macrophages and improves response to $\mathrm{T}$ cell checkpoint immuno-therapy in pancreatic cancer models. Cancer Res. 2014;74(18):5057-69. [PubMed: 25082815]

32. Wainberg ZA, Piha-Paul SA, Luke JJ et al. First-inhuman phase 1 dose escalation and expansion of a novel combination, anti-CSF-1 receptor (cabiralizumab) plus anti-PD-1 (nivolumab), in patients with advanced solid tumors. Presented at: 32nd SITC Annual Meeting; November 8-12, 2017; National Harbor, MD Abstract O4. 
33. Calvo A, Joensuu H, Sebastian M, et al. Phase Ib/II study of lacnotuzumab (MCS110) combined with spartalizumab (PDR001) in patients (pts) with advanced tumors. J Clin Oncol. 2018;36(15):S3014.

34•. Cui R, Yue W, Lattime E, et al. Targeting tumor-associated macrophages to combat pancreatic cancer. Oncotarget. 2016;7(31):50735-54. [PubMed: 27191744] A comprehensive review detailing the double-edged role of macrophages in both promoting tumorigenesis and limiting the process. The article delves into the role of cytokines such as CCL2 and CCL5 in generating tumor promoting macrophages (Th2).

35. Zhu Y, Herndon J, Sojka D, et al. Tissue-resident macrophages in pancreatic ductal adenocarcinoma originate from embryonic hematopoiesis and promote tumor progression. Immunity. 2017;47(2):323-38. [PubMed: 28813661]

36. Jiang H, Hedge S, Knolhoff B, et al. Targeting focal adhesion kinase renders pancreatic cancers responsive to checkpoint immunotherapy. Nat Med. 2016;22:851-60. [PubMed: 27376576]

37. Wang-Gillam A, Lockhart C, Tan B, et al. Phase I study of defactinib combined with pembrolizumab and gemcitabine in patients with advanced cancer. J Clin Oncol. 2018;36(4):S380.

38. Rastelli L, Gupta S, Jagga Z, et al. Efficacy and immune modulation by BXCL701 a dipeptidyl peptidase inhibitor, NKTR-214 a CD122-biased immune agonist with PD1 blockade in murine pancreatic tumors. J Clin Oncol. 2018;36(15):S3085.

39. Friberg M, Nielsen B, Mortensen MB, et al. Key players in pancreatic cancer-stroma interaction: Cancer-associated fibroblasts, endothelial and inflammatory cells. World J Gastroenterol. 2016;22(9):2678-700. [PubMed: 26973408]

40. Ping L, Ding N, Shi Y, Feng L, Li J, Liu Y, et al. The Bruton's tyrosine kinase inhibitor ibrutinib exerts immunomodulatory effects through regulation of tumor-infiltrating macrophages. Oncotarget. 2017;8(24):39218-29. [PubMed: 28424405]

41. Tempero M, Coussens L, Fong L, et al. A randomized, double-blind, placebo-controlled study of ibrutinib, a Bruton tyrosine kinase inhibitor, with nab-paclitaxel and gemcitabine in the first-line treatment of patients with metastatic pancreatic adenocarcinoma (RESOLVE). J Clin Oncol. 2016;34(15):S2601.

42. Hong D, Rasco D, Veeder M, et al. A multicenter study of the Bruton's tyrosine kinase (BTK) inhibitor ibrutinib plus durvalumab in patients with relapsed/refractory $(\mathrm{R} / \mathrm{R})$ solid tumors. J Clin Oncol. 2018;36(15):S2578.

43. Xia X, Wu W, Huang C, Cen G, Jiang T, Cao J, et al. SMAD4 and its role in pancreatic cancer. Tumour Biol. 2015;36(1):111-9. [PubMed: 25464861]

44. Ahmed S, Bradshaw AD, Gera S, Dewan M, Xu R. The TGF- $\beta /$ Smad4 signaling pathway in pancreatic carcinogenesis and its clinical significance. J Clin Med. 2017;6(1):5.

45. Melisi D, Garcia-Carbonero R, Macarulla T, et al. A phase II, double-blind study of galunisertib +gemcitabine (GG) vs gemcitabine+placebo (GP) in patients (pts) with unresectable pancreatic cancer (PC). J Clin Oncol. 2016;34(15):S4019.

46. Papadopolous K, Naing A, Infante J, et al. Anti-tumor activity of PEGylated human IL-10 (AM0010) in patients with pancreatic or colorectal cancer. J Clin Oncol. 2016;34(15):S3082.

47. Hecht JR, Naing A, Falchook G, et al. Phase 1b study with PEGylated human IL-10 (AM0010) with 5-FU and oxaliplatin (FOLFOX) in metastatic pancreatic adenocarcinoma (PDAC). J Clin Oncol. 2017;35(4):S399.

48. Jin D, Fan J, Wang L, Thompson LF, Liu A, Daniel BJ, et al. CD73 on tumor cells impairs antitumor T-cell responses: a novel mechanism of tumor-induced immune suppression. Cancer Res. 2010;70(6):2245-55. [PubMed: 20179192]

49. Overman M, LoRusso P, Strickler J, et al. Safety, efficacy and pharmacodynamics (PD) of MEDI9447 (oleclumab) alone or in combination with durvalumab in advanced colorectal cancer (CRC) or pancreatic cancer (panc). J Clin Oncol. 2018;suppl:abstr 4123.

50. Pendergrast G, Malachowski W, DuHadaway J, et al. Discovery of IDO1 inhibitors: from bench to bedside. Cancer Res. 2017;77(24):6795-811. [PubMed: 29247038]

51. Zhang T, Tan XL, Xu Y, Wang ZZ, Xiao CH, Liu R. Expression and prognostic value of indoleamine 2,3-dioxygenase in pancreatic cancer. Chin Med J. 2017;130(6):710-6. [PubMed: 28303855] 
52. Bahary N, Garrido-Laguna I, Wang-Gillam A, et al. Results of the phase Ib portion of a phase I/II trial of the indoleamine 2,3-dioxygenase pathway (IDO) inhibitor indoximod plus gemcitabine/ nab-paclitaxel for the treatment of metastatic pancreatic cancer. J Clin Oncol. 2016;34(4):S452.

53. Lum L, Choi M, Thakur A, et al. Five advanced pancreatic cancer patients in a phase I study of anti-CD3 $\mathrm{x}$ anti-EGFR bispecific antibody armed activated T cells (BATS). J Immunother Cancer. 2015;3(Suppl 2):P55.

54. Salnikov AV, Groth A, Apel A, Kallifatidis G, Beckermann BM, Khamidjanov A, et al. Targeting of cancer stem cell marker EpCAM by bispecific antibody EpCAMxCD3 inhibits pancreatic carcinoma. J Cell Mol Med. 2009;13(9B):4023-33. [PubMed: 20196789]

55. Cioffi M, Dorado J, Baeurle PA, et al. EpCAM/CD3-bispecific T-cell engaging antibody MT110 eliminates primary human pancreatic cancer stem cells. Clin Cancer Res. 2012;8(2):465-74.

56. Heiss MM, Murawa P, Koralewski P, et al. The trifunctional antibody catumaxomab for the treatment of malignant ascites due to epithelial cancer: results of a prospective randomized phase II/III trial. Int J Cancer. 2010;27(9):2209-21. 


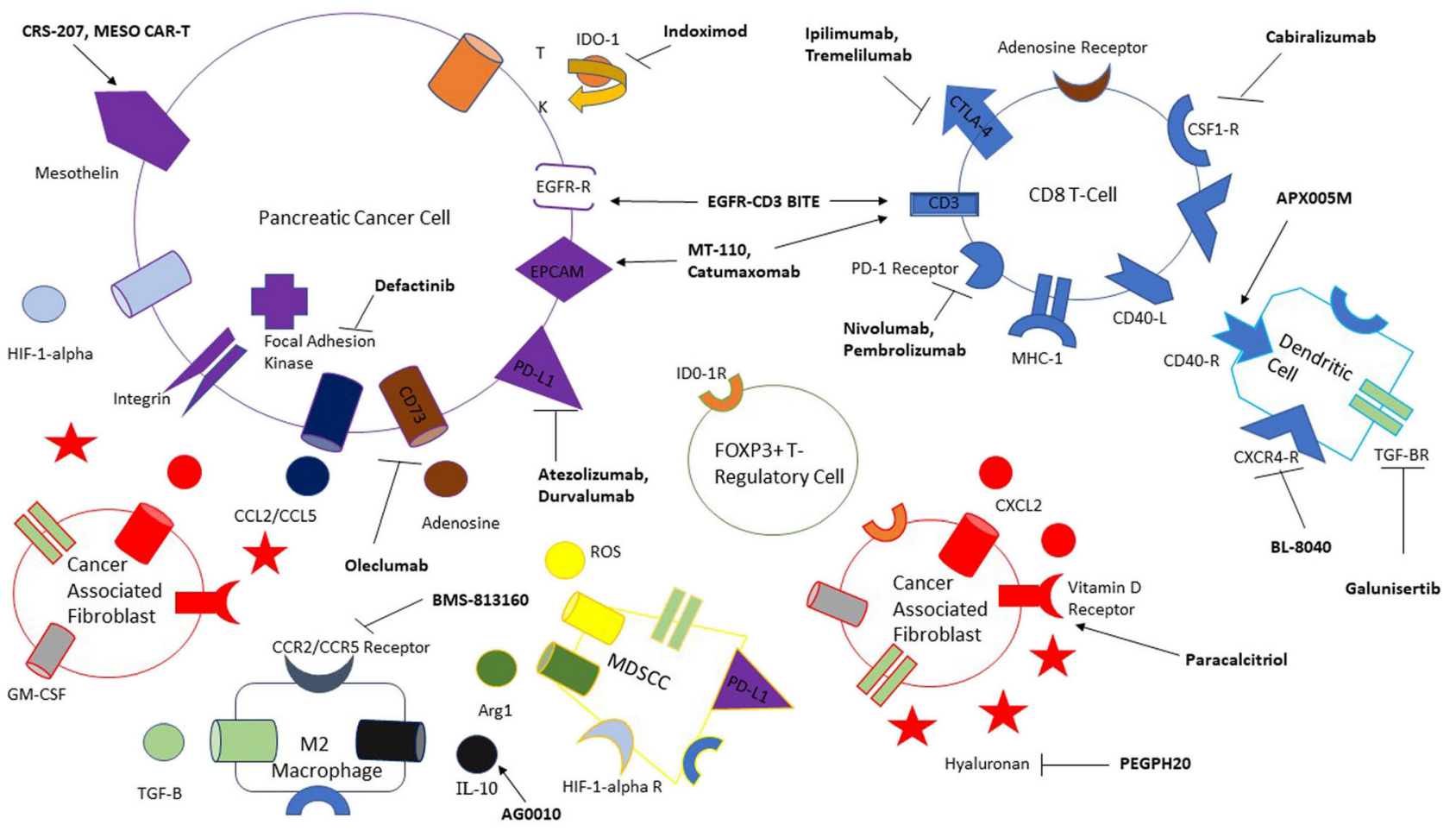

Fig. 1.

The immune mileu comprising the pancreatic cancer tumor microenvironment. Drugs targeting different receptors, cytokines, or stomal components are bolded in black. An arrow pointing toward a receptor implies an agonist while an inhibitor is indicated by the-I symbol. Unlabeled same-shape receptors on different cells imply the same receptor as originally labeled. $\mathrm{T}$ tryptophan, $\mathrm{K}$ kynurenine. 